\title{
Versatile Polarization Generation and Manipulation Using Dielectric Metasurfaces
}

Ding, Fei; Chang, Bingdong; Wei, Qunshuo; Huang, Lingling; Guan, Xiaowei; Bozhevolnyi, Sergey I.

Published in:

Laser and Photonics Reviews

Link to article, DOI:

10.1002/lpor.202000116

Publication date:

2020

Document Version

Peer reviewed version

Link back to DTU Orbit

Citation (APA):

Ding, F., Chang, B., Wei, Q., Huang, L., Guan, X., \& Bozhevolnyi, S. I. (2020). Versatile Polarization Generation and Manipulation Using Dielectric Metasurfaces. Laser and Photonics Reviews, 14(11), [2000116]. https://doi.org/10.1002//por.202000116

\section{General rights}

Copyright and moral rights for the publications made accessible in the public portal are retained by the authors and/or other copyright owners and it is a condition of accessing publications that users recognise and abide by the legal requirements associated with these rights.

- Users may download and print one copy of any publication from the public portal for the purpose of private study or research.

- You may not further distribute the material or use it for any profit-making activity or commercial gain

- You may freely distribute the URL identifying the publication in the public portal 


\section{Arbitrary Polarization Generation and Manipulation Using Dielectric Metasurfaces}

Fei Ding, ${ }^{1, * \dagger}$ Bingdong Chang, ${ }^{2,}{ }^{*}$ Lingling Huang, ${ }^{3}$ Xiaowei Guan, ${ }^{4}$ and Sergey I. Bozhevolnyi ${ }^{1, \dagger}$

${ }^{1}$ Centre for Nano Optics, University of Southern Denmark, Campusvej 55, DK-5230 Odense, Denmark

${ }^{2}$ DTU Danchip, Technical University of Denmark, Kongens Lyngby 2800, Denmark ${ }^{3}$ School of Optics and Photonics, Beijing Institute of Technology, Beijing 100081, China

${ }^{4}$ DTU Fotonik, Department of Photonics Engineering, Technical University of Denmark, 2800 Kgs. Lyngby, Denmark

*These authors contributed equally to this work.

${ }^{\dagger}$ Corresponding author. Email: feid@mci.sdu.dk (F.D.); seib@mci.sdu.dk (S.I.B.)

\section{ORCID}

Fei Ding: 0000-0001-7362-519X

Lingling Huang: 0000-0002-3647-2128

Sergey I. Bozhevolnyi: 0000-0002-0393-4859 


\begin{abstract}
:
Advances in polarization optics and integrated photonics require fundamentally new polarization-modulation strategies capable of arbitrarily generating and fully manipulating the state of polarization with ultracompact footprints, surface-confined configurations, and unprecedentedly large bandwidths. We demonstrate how dielectric metasurfaces can be leveraged to produce arbitrary polarization states with controllable wavefronts all from a linearly-polarized light source. Advanced polarization-manipulating meta-polarizers, including polarization-resolved multifocal metalens and vectorial holographic display, have been realized using judiciouslydesigned dielectric metasurfaces composed of segmented sub-arrays able to manipulate, simultaneously and independently, the polarization and phase of the transmitted beams. The versatility of our concept provides opportunities to develop a complete set of flat polarization optics for integrated photonics and quantum optics.
\end{abstract}

One Sentence Summary: We demonstrate how dielectric metasurfaces can be leveraged to produce arbitrary polarization states with controllable wavefronts all from a linearly-polarized light source. 


\section{Main Text:}

Polarization is one of the intrinsic properties of light carrying valuable information in fundamental sciences and practical applications (1). Conventional polarization optics use birefringence to generate or manipulate the state of polarization (SOP), where the phase retardation between two orthogonally-polarized components is gradually accumulated as light propagates over a distance much longer than its wavelength. Therefore, the resulting polarization optics are inherently bulky and voluminous, going against the recent trends of integration and miniaturization in photonics, which in turn boosts the developments of metasurfaces that revolutionize optical designs by replacing traditional bulky optical components with ultrathin planar meta-devices (25). Due to the unprecedented capabilities of molding light, metasurfaces have been extensively explored to demonstrate ultra-compact devices with ultimate low-profiles that enable polarization conversion (6-9) and polarization detection (10-13). However, these metasurface-based polarization converters are always designed to perform a single functionality; in case of a half-wave plate (HWP), it can only yield linear-tolinear (or circular-to-circular) polarization transformation for plane wave (7-9). Ideally, one would prefer a versatile polarization generator capable of generating arbitrarily well-defined SOPs, and simultaneously manipulating the corresponding output wavefronts at will, while exhibiting the advantages of planar profiles, compactness, and relative ease of fabrication.

By contrast, polarization generators that produce multiple SOPs from a linearlypolarized (LP) light have been demonstrated with gap-plasmon metasurfaces $(14,15)$ and the dielectric metasurface grating (16). However, the plasmonic meta-devices are constrained to operate in reflection while the diatomic metasurface (15) only works properly under oblique incidence, thereby hindering their potential applications. Additionally, the efficiency is limited even though the loss of metal is ignored. For instance, the theoretical upper bound of the efficiency for a certain SOP generated in the desired direction is $50 \%$ since there are two identical beams produced in opposite directions (14). For diatomic meta-atoms, the enlarged displacement between two orthogonal meta-atoms results in a decrease of the diffraction efficiency due to the lower scattering efficiency of sparsely distributed meta-atoms (15). Regarding the dielectric metasurface grating, the total diffraction efficiency on a set of defined diffraction orders that produce multiple SOPs is limited to a value of $\sim 23.61$ (16). 
Here, we generalize the idea of a dielectric metasurface polarizer (meta-polarizer) that overcomes the aforementioned limitations and performs arbitrary polarization transformation from an LP light to six beams with arbitrarily specified SOPs and wavefronts in the near-infrared range. We refer to this as arbitrary polarization generation and manipulation because there are no restrictions on output SOPs and wavefronts, superior to previous demonstrations that only produce planar wavefronts $(14,16)$. We implement this design by using segmented sub-arrays composed of amorphous silicon $(\alpha-\mathrm{Si})$ meta-atoms that function as nanoscale wave plates (nanoWPs) with, simultaneously and independently, engineered phase and polarization responses. Capitalizing on the meta-polarizers, polarization-resolved multifocal metalens and vectorial holographic display are experimentally demonstrated.

The working principle of the proposed meta-polarizer that generates and manipulates multiple beams possessing distinct SOPs and wavefronts from an $x$ polarized incident beam is schematically illustrated in Fig. 1A, which consists of six segmented sub-arrays that each occupies a $60^{\circ}$ circular sector (Fig. 1B), being hereby incorporated in a circular configuration. Each sector-shaped sub-array comprises $\alpha$-Si meta-atoms tailored to impart particular polarization transformation and phase shifts simultaneously for one of the six specified SOPs [i.e. $|x\rangle,|y\rangle,|a\rangle,|b\rangle,|r\rangle$ and $|l\rangle$ states], which represent horizontal/vertical linear polarizations, $\pm 45^{\circ}$ linear polarizations, and right-handed/left-handed circular polarizations, respectively. It should be noted that the available SOPs are obviously not limited to this particular demonstration. In fact, this meta-polarizer is able to realize arbitrary SOPs and wavefronts.

The building blocks are $\alpha$-Si meta-atoms of rectangular or ellipse cross-sections in a square lattice atop a fused silica substrate (Fig. 1B, right panel). By tailoring the shapes, dimensions, and orientations, we can fully and independently control the polarization and phase (i.e. $2 \pi$ phase range) of the transmitted light while maintaining high transmission efficiency when the induced dielectric and magnetic dipole resonances are spectrally overlapped at the design wavelength of $\lambda_{d}=850 \mathrm{~nm}$ (fig. S2) (5,17-20). As such, each meta-atom acts as a polarization-generating phase modulator that maps the input LP plane wave to complicated output wavefronts with arbitrary SOPs. Under the excitation of an $x$-polarized light, the output beam passing through the meta-polarizer can be expressed by the Jones Matrix (see supplementary text): 


$$
E_{\text {out }}=|t| e^{i \varphi_{x 0}}\left[\begin{array}{c}
\cos ^{2} \theta+e^{i \delta} \cdot \sin ^{2} \theta \\
\left(1-e^{i \delta}\right) \cdot \frac{\sin 2 \theta}{2}
\end{array}\right]
$$

where $|t|$ is the transmission amplitude, $\theta$ is the in-plane orientation angle, $\varphi_{x 0}$ and $\varphi_{y 0}$ are the phase shifts for $x$ - and $y$-polarized light, and $\delta=\varphi_{y 0}-\varphi_{x 0}$. This matrix provides a general mapping from $|x\rangle$ state to arbitrary SOPs with extra degrees of freedom to modulate output wavefronts, in contrast to previous SOPs generators that only have planar wavefront outputs $(14,16)$.

For isotropic meta-atoms (i.e. $\delta=0$ ) with any value of $\theta$, the output is always $|x\rangle$ state but gains an additional phase shift $\varphi_{\mathrm{x} 0}$. In Fig. 1C, we plot the transmission and phase shift of six isotropic meta-atoms comprising the sub-array for $|x\rangle$ state generation and manipulation at $\lambda_{\mathrm{d}}=850 \mathrm{~nm}$, which exhibit high transmission and provide an incremental phase of $\pi / 3$ between adjacent meta-atoms for the co-polarized transmitted light (fig. S3A). If the meta-atoms are anisotropic that function as nanoHWPs, $\delta=\pi$, the output field becomes linearly polarized and the angle of linear polarization is $2 \theta$ :

$$
E_{\text {out }}=|t| e^{i \varphi_{x 0}}\left[\begin{array}{l}
\cos 2 \theta \\
\sin 2 \theta
\end{array}\right]
$$

In this way, we can generate any linear polarization with spatially-orientated nanoHWPs. For instance, $|y\rangle,|a\rangle,|b\rangle$ states can be accordingly produced when the orientation angle $\theta$ is set to be $45^{\circ}, 22.5^{\circ}$, and $67.5^{\circ}$, respectively (fig. S4). The simulated transmission and phase shift in Fig. 1D shows that each meta-atom works properly as a highly-efficient nano-HWP at $\lambda_{\mathrm{d}}=850 \mathrm{~nm}$, where the cross-polarized transmission $T_{\mathrm{yx}}$ is above $82 \%$ while the co-polarized transmission $T_{\mathrm{xx}}$ is greatly suppressed. Moreover, the additional phase term allows us to engineer the output wavefronts, superior to conventional metasurface-based HWPs (9). Therefore, we can independently control the phase of cross-polarized fields and eventually achieve the phase-gradient for further wavefront engineering (fig. S3B). Apart from linearpolarization generation, circular or elliptical polarizations can be accordingly generated and modulated with anisotropic meta-atoms possessing the functionality of quarter-wave plates (QWPs) where $\delta$ is equal to $\pm \pi / 2$. In this case, the output field is elliptically-polarized:

$$
E_{\text {out }}=|t| e^{i \varphi_{x 0}}\left[\begin{array}{c}
\cos ^{2} \theta \pm i \cdot \sin ^{2} \theta \\
(1 \mp i) \cdot \frac{\sin 2 \theta}{2}
\end{array}\right]
$$


Once the orientation angle $\theta$ is $45^{\circ}$, circular polarizations can be created:

$$
E_{\text {out }}=|t| \frac{1 \pm i}{2} e^{i \varphi_{x 0}}\left[\begin{array}{c}
1 \\
\mp i
\end{array}\right]
$$

To maintain good performance, three highly-efficient nano-QWPs with a phase step of $2 \pi / 3$ are used to construct the sub-array for circular polarization generation and manipulation (Fig. 1E and fig. S3C). Here it is worth noting that more phase levels don't necessarily ensure higher efficiencies for QWPs since the increased variations in efficiencies and operating bandwidths will, in turn, affect the performance. As the numbering changes from 1 to 3 , the phase shift of the transmitted wave increases linearly from 0 to $2 \pi / 3$ in the slow axis and from $\pi / 2$ to $7 \pi / 6$ in the fast axis, respectively, while the phase differences between the two axes maintain $\pi / 2$ (Fig. 1E). Therefore, $|r\rangle$ and $|l\rangle$ states can be generated and fully controlled with this group of QWP meta-atoms.

To validate the versatility and high-performance of the meta-polarizers, we first demonstrate a polarization-resolved multifocal metalens that focuses an $x$-polarized beam to six different spots with distinct and well-defined SOPs (21). In our design, six foci are all separated from the center point with a distance of $s=20 \mu \mathrm{m}$. Fig. 2, A and $B$ to $D$ show the optical and scanning electron microscope (SEM) images of a metalens sample with a diameter of $D=100 \mu \mathrm{m}$ and a focal length of $f=90 \mu \mathrm{m}$ at $\lambda_{\mathrm{d}}$ $=850 \mathrm{~nm}$, fabricated by patterning a 500-nm-thick $\alpha$-Si film on a fused silica substrate (21). The fabricated metalens was characterized using a custom-built optical setup that allows to continuously map intensity distributions at different $x-y$ planes along the $z$-direction (fig. S6). The left panel of Fig. 2E shows the two-dimensional intensity slices along $z$-direction without any polarization analyzer placed in front of the CCD camera when the sample is illuminated with an $x$-polarized Gaussian beam at normal incidence. At the measured focal plane of $z=89 \mu \mathrm{m}$, six diffraction-limited spots are formed with negligible backgrounds, indicating excellent phase realization and high-efficiency of each meta-atom. To determine the SOPs, additional polarizers and WPs were added. The polarization-resolved intensity profiles in different polarization base are plotted in Fig. 2E (right panel), which shows $>20 \mathrm{~dB}$ extinction ratios for all generated SOPs (fig. S7). Impressively, the experimentally reconstructed Stokes parameter (see supplementary text) replicate reasonably well the theoretical predictions on the Poincaré sphere. The average two-norm error between the reconstructed and theoretical Stokes vector for all six spots is as small as $6.07 \%$ at $\lambda_{\mathrm{d}}$ 
$=850 \mathrm{~nm}$. More experimental results at other wavelengths are listed in figs. S8 and S9, validating the excellent performance of the fabricated metalens in terms of accurate SOPs generation, good focusing capability and large bandwidth. In the wavelength range of $800-900 \mathrm{~nm}$, the experimental Stokes parameters closely represent theoretical values with the average deviation of all six spots below 10\% (Fig. 2G). However, when the wavelength moves further away from $\lambda_{\mathrm{d}}$ (i.e. $\lambda=750$ and 1050 $\mathrm{nm})$, the averaged discrepancy increases, which is mainly ascribed to the deteriorated performance of nano-QWPs that results in large deviations from Spot $i i$ and $v$. Although the retrieved Stokes parameters show larger discrepancies at $\lambda=750$ and $1050 \mathrm{~nm}$, the metalens maintains excellent focusing capability and the measured extinction ratio of a given SOP is still high (fig. S7). We also measured the focusing efficiency of the metalens. As shown in Fig. $2 \mathrm{H}$, the fabricated metalens has a measured efficiency of $64.6 \%$ at $\lambda_{\mathrm{d}}=850 \mathrm{~nm}$, which is slightly lower than the theoretical upper limit of $85.41 \%(21)$. We attribute this deviation to fabrication imperfections and nonnegligible coupling between adjacent meta-atoms. In spite of the attendant reduction in measured efficiency, it is remarkable that the efficiency remains above 50\% within the wavelength range of 800-1000 nm.

In addition to producing multiple focused beams with arbitrary SOPs, the metapolarizer allows us to realize the most complex and general wavefront shaping, namely holography, while maintaining the capability of spatially manipulating SOPs, thereby demonstrating vectorial holography. Fig. 3A illustrates the concept of vectorial holographic display that reconstructs the Chinese lucky cloud image with arbitrarily specified polarization distributions in the far-field based on the GerchbergSaxton algorithm (22). Similarly, the lucky cloud is also divided into six sub-images represented by corresponding metasurface-sectors, which are independently encoded with different SOPs (Fig. 3, B and C). We first illuminated one single sector with an $x$-polarized light and conducted far-field measurements (fig. S10). From Fig. 3, D to I, one can clearly see a vivid and high-fidelity sub-image reconstructed spatially in the far-field, which corresponds to the illuminated sector and exhibits a specified SOP with high extinction ratio to its counterpart. For instance, sub-image $i$ is totally suppressed while sub-image $i i$ gets pronounced when the output polarization is varied from $|x\rangle$ to $|y\rangle$ state (Fig. 3, D and E). Similar phenomena can be observed for other sub-images with different output polarizations (Fig. 3, F to I). We measured about 51\% hologram efficiencies for the reconstructed sub-images with linear polarizations at $\lambda_{\mathrm{d}}$ 
$=850 \mathrm{~nm}$, a little higher than the value of $\sim 41 \%$ for the sub-images with circular polarizations, which results from fewer phase levels of nano-QWPs that cause twin images and more scattering loss. We further carried out verification of polarizationresolved multiplexed holograms with $x$-polarized excitation. Without any polarization analyzers, the complete lucky cloud with good fidelity was obtained, regardless of the noticeable zero-order transmission due to fabrication imperfections and the gap between adjacent sectors (Fig. 3J). Once the analyzers are added, specific images with distinguished patterns can be reconstructed accordingly under certain SOPs, indicating the potential for multistate encrypted anticounterfeiting. We also tested the performance of our meta-hologram at other wavelengths and found it exhibits relatively good performance with a relatively large working bandwidth in generating versatile polarizations and projecting unique holograms simultaneously (figs. S11 and S12).

Our design shows a general metasurface-mediated mapping between a linear polarization input and versatile polarization outputs and can produce arbitrary SOPs with distinct wavefronts by employing all dielectric meta-atoms that function as nanoWPs with controllable phase and polarization responses, thereby mimicking the functionalities of cascaded polarization components. Capitalizing on this concept, we have demonstrated polarization-resolved multifocal metalens and vectorial holographic display over a broadband infrared spectrum range. Due to the compact nature, such meta-polarizers enable easy integration and fancy applications in polarization optics. Moreover, the spatial- and polarization-resolved meta-polarizer could emerge as an essential component for quantum nanophotonics when interacting with nonclassical optical fields at the single-photon level (23-25). 

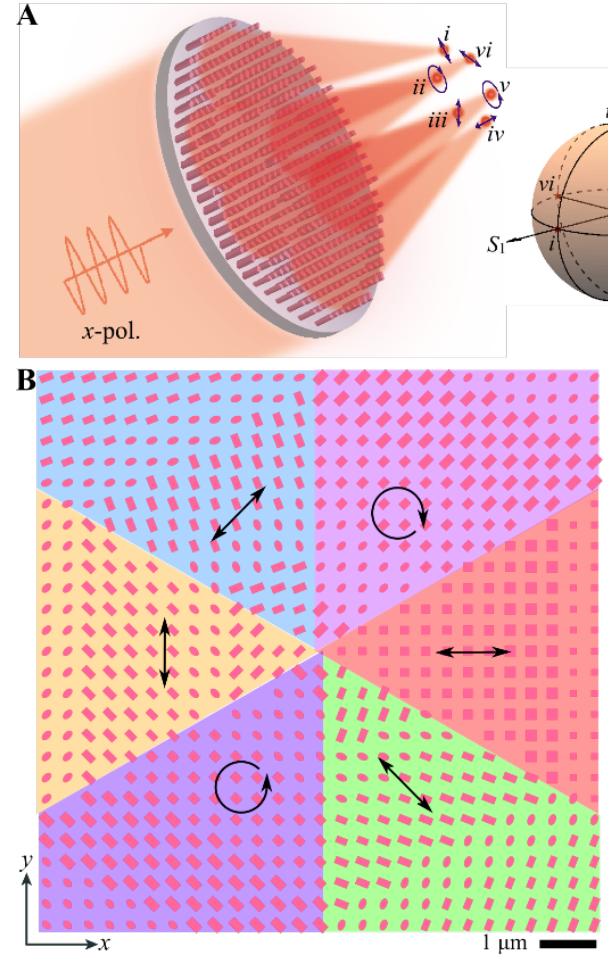
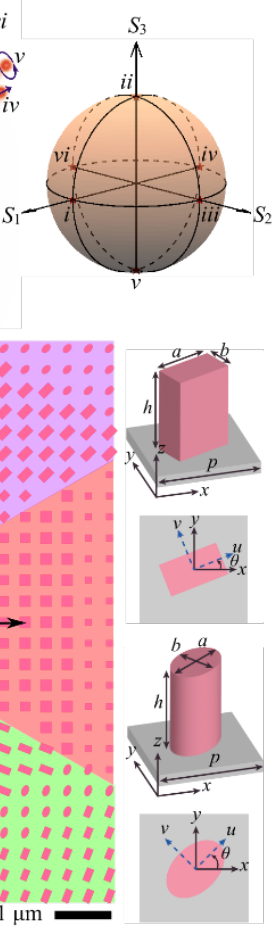
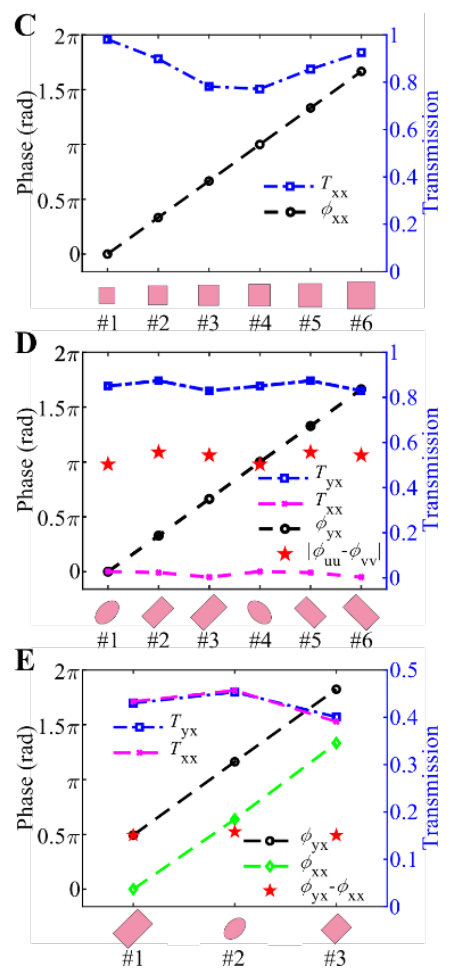

Fig. 1. Arbitrary polarization generation and manipulation. (A) Schematic of the concept for arbitrary polarization generation and manipulation with a dielectric metapolarizer, which converts an $x$-polarized beam into six beams with arbitrarily specific SOPs and wavefronts. (B) Schematic of the central part of a typical design used to carry out the function in (A). The background colors represent the sub-arrays and the polarization basis in each part is shown with a black arrow. The $\alpha$-Si meta-atom unit cell has the height of $h=500 \mathrm{~nm}$ and the lattice constant of $p=360 \mathrm{~nm}$. (C) Simulated transmission and phase shift of the six meta-atoms comprising the subarray to generate and manipulate the $|x\rangle$ state at $\lambda_{\mathrm{d}}=850 \mathrm{~nm}$. (D) Simulated transmission and phase shift of the six meta-atoms comprising the sub-arrays to generate and manipulate the $|a\rangle,|y\rangle$, and $|b\rangle$ states at $\lambda_{\mathrm{d}}=850 \mathrm{~nm}$. (E) Simulated transmission and phase shift of the three meta-atoms comprising sub-arrays to generate and manipulate the $|r\rangle$ and $|l\rangle$ states at $\lambda_{\mathrm{d}}=850 \mathrm{~nm}$. 

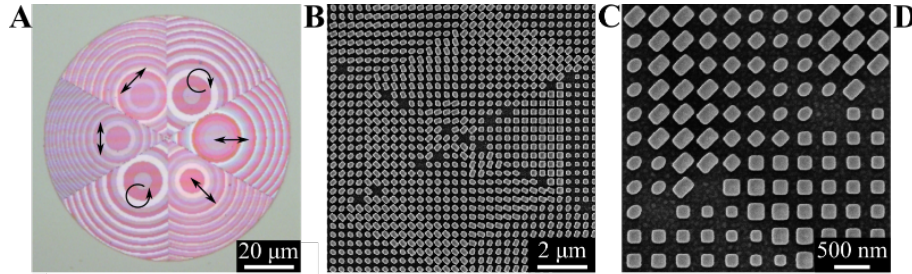

$\mathbf{E}$

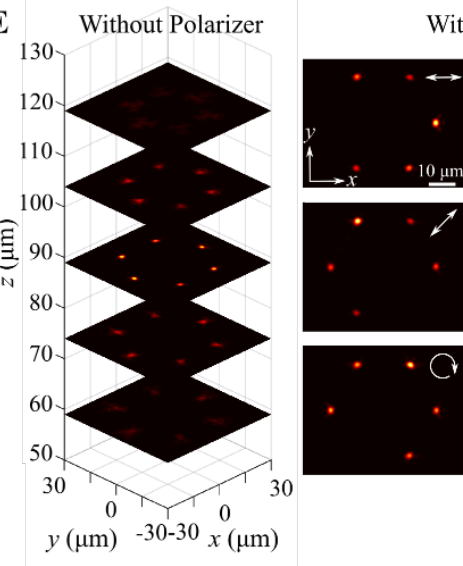

With Polarizer

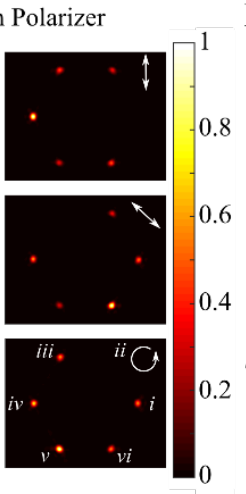

F

G
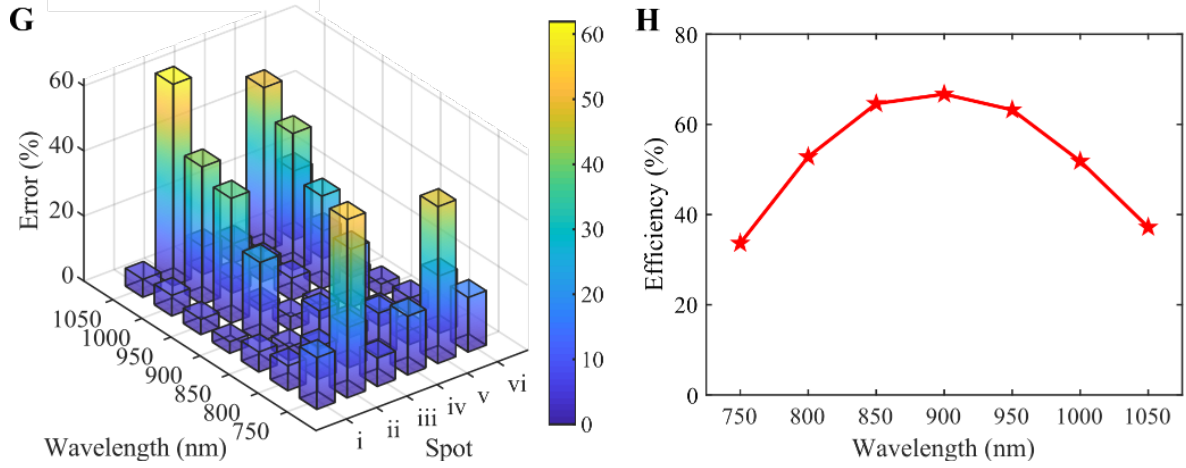

Fig. 2. Experimental demonstration of the polarization-resolved multifocal metalens. (A) Optical image of the fabricated metalens for all-polarization generation and focusing. (B to D) SEM image of the fabricated metalens. (E) Optical characterization of the metalens at $\lambda_{\mathrm{d}}=850 \mathrm{~nm}$ for $x$-polarized incident light. Left (E): Focal spots evolution along the $z$-axis without any polarization analyzer. Right (E): Polarization-resolved intensity profiles at the focal plane. (F) The theoretical (blue circle) and experimentally reconstructed (red stars) Stokes parameters on the Poincaré sphere at $\lambda_{\mathrm{d}}=850 \mathrm{~nm}$. (G) Two-norm error between the theoretical and reconstructed Stokes parameters of the six focal spots as a function of wavelength. (H) Measured focusing efficiency of the metalens as a function of wavelength. 


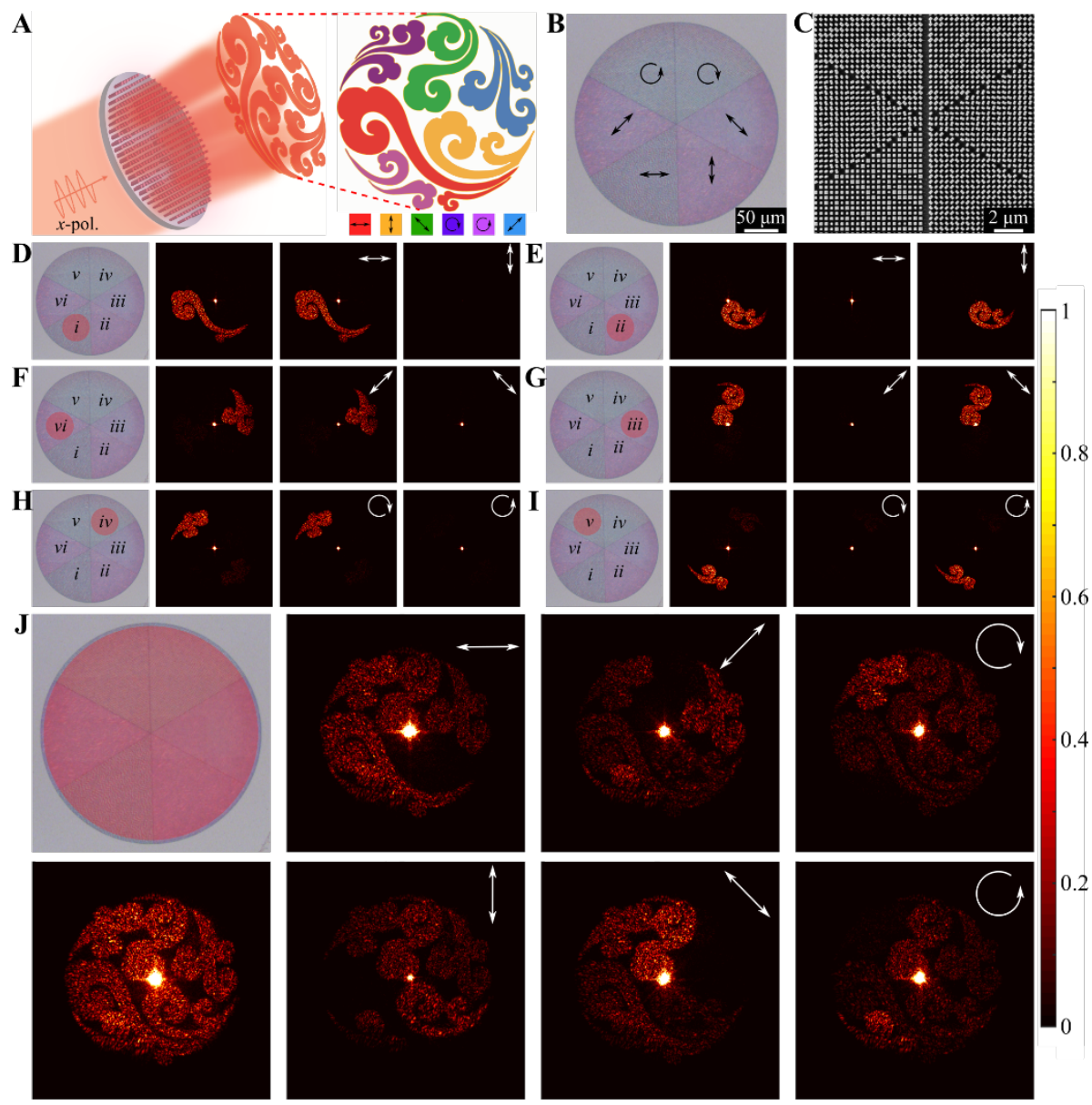

Fig. 3. Experimental demonstration of the meta-hologram for vectorial holographic display. (A) Schematic of the concept for vectorial holographic display, where the holographic image with spatially-varying SOPs can be generated with an $x$ polarized light. The different colors and black arrows represent different SOPs. (B) Optical image of the fabricated meta-hologram. (C) SEM image of the fabricated meta-hologram. (D to I) Measured holographic images by illuminating one sector of the meta-hologram at $\lambda_{\mathrm{d}}=850 \mathrm{~nm}$. (J) Measured holographic images by illuminating the whole meta-hologram at $\lambda_{\mathrm{d}}=850 \mathrm{~nm}$. In ( $\mathrm{D}$ to $\mathrm{J}$ ), the incident light is $x$-polarized and the white arrow represents the output polarization state when analyzers are added. 


\section{References and notes:}

1. M. Born, E. Wolf, Principle of Optics, 7th ed. (Cambridge University, 1999).

2. V. Kildishev, A. Boltasseva, V. M. Shalaev, Planar photonics with metasurfaces. Science 339, 1232009 (2013).

3. N. F. Yu, F. Capasso, Flat optics with designer metasurfaces. Nat. Mater. 13, 139150 (2014).

4. F. Ding, A. Pors, S. I. Bozhevolnyi, Gradient metasurfaces: a review of fundamentals and applications. Rep. Prog. Phys. 81, 026401 (2018).

5. S. M. Kamali, E. Arbabi, A. Arbabi, and A. Faraon, A review of dielectric optical metasurfaces for wavefront control. Nanophotonics 7, 1041-1068 (2018).

6. N. Yu, F. Aieta, P. Genevet, M. A. Kats, Z. Gaburro, F. Capasso, A broadband, background-free quarter-wave plate based on plasmonic metasurfaces. Nano Lett. 12, 6328-6333 (2012).

7. N. K. Grady, J. E. Heyes, D. R. Chowdhury, Y. Zeng, M. T. Reiten, A. K. Azad, A. J. Taylor, D. A. Dalvit, H. T. Chen, Terahertz metamaterials for linear polarization conversion and anomalous refraction. Science 340, 1304 (2013).

8. F. Ding, Z. Wang, S. He, V. M. Shalaev, A. V. Kildishev, Broadband highefficiency half-wave plate: a supercell-based plasmonic metasurface approach. ACS Nano 9, 4111-4119 (2015).

9. S. Kruk, B. Hopkins, I. I. Kravchenko, A. Miroshnichenko, D. N. Neshev, Y. S. Kivshar, Broadband highly efficient dielectric metadevices for polarization control. APL Photon. 1, 030801 (2016).

10. A. Pors, M. G. Nielsen, S. I. Bozhevolnyi, Plasmonic metagratings for simultaneous determination of Stokes parameters. Optica 2, 716-723 (2015).

11. E. Maguid, I. Yulevich, D. Veksler, V. Kleiner, M. L. Brongersma, E. Hasman, Photonic spin-controlled multifunctional shared-aperture antenna array. Science 352, 1202-1206 (2016).

12. F. Ding, A. Pors, Y. Chen, V. A. Zenin, S. I. Bozhevolnyi, Beam-size-invariant spectropolarimeters using gap-plasmon metasurfaces. ACS Photonics 4, 943-949 (2017).

13. E. Arbabi, S. M. Kamali, A. Arbabi, A. Faraon, Full-stokes imaging polarimetry using dielectric metasurfaces. ACS Photonics 5, 3132-3140 (2018). 
14. P. C. Wu, W.-Y. Tsai, W. T. Chen, Y.-W. Huang, T.-Y. Chen, J.-W. Chen, C. Y. Liao, C. H. Chu, G. Sun, D. P. Tsai, Versatile polarization generation with aluminum plasmonic metasurface. Nano Lett. 17, 445-452 (2017).

15. Z.-L. Deng, J. Deng, X. Zhuang, S. Wang, K. Li, Y. Wang, Y. Chi, X. Ye, J. Xu, G. P. Wang, R. Zhao, X. Wang, Y. Cao, X. Cheng, G. Li, X. Li, Diatomic metasurface for vectorial holography. Nano Lett. 18, 2885-2892 (2018).

16. N. A. Rubin, A. Zaidi, M. Juhl, R. P. Li, J. P. B. Mueller, R. C. Devlin, K. Leósson, F. Capasso, Polarization state generation and measurement with a single metasurface. Opt. Express 26, 21455-21478 (2018).

17. D. Lin, P. Fan, E. Hasman, M. L. Brongersma, Dielectric gradient metasurface optical elements. Science 345, 298-302 (2014).

18. Arbabi, Y. Horie, M. Bagheri, A. Faraon, Dielectric metasurfaces for complete control of phase and polarization with subwavelength spatial resolution and high transmission. Nat. Nanotechnol. 10, 937-943 (2015).

19. M. Khorasaninejad, W. T. Chen, R. C. Devlin, J. Oh, A. Y. Zhu, F. Capasso, Metalenses at visible wavelengths: Diffraction-limited focusing and subwavelength resolution imaging. Science 352, 1190-1194 (2016).

20. I. Kuznetsov, A. E. Miroshnichenko, M. L. Brongersma, Y. S. Kivshar, B. Luk'yanchuk, Optically resonant dielectric nanostructures. Science 354, aag2472 (2016).

21. See supplementary materials and methods.

22. R. Zhao, B. Sain, Q. Wei, C. Tang, X. Li, T. Weiss, L. Huang, Y. Wang, T. Zentgraf, Multichannel vectorial holographic display and encryption. Light Sci. Appl. 7, 95 (2018).

23. J. Wang, S. Paesani, Y. Ding, R. Santagati, P. Skrzypczyk, A. Salavrakos, J. Tura, R. Augusiak, L. Mančinska, D. Bacco, D. Bonneau, J. W. Silverstone, Q. Gong, A. Acín, K. Rottwitt, L. K. Oxenløwe, J. L. O’Brien, A. Laing, M. G. Thompson, Multidimensional quantum entanglement with large-scale integrated optics. Science 360, 285-291 (2018).

24. T. Stav, A. Faerman, E. Maguid, D. Oren, V. Kleiner, E. Hasman, M. Segev, Quantum entanglement of the spin and orbital angular momentum of photons using metamaterials. Science 361, 1104-1108 (2018).

25. K. Wang, J. G. Titchener, S. S. Kruk, L. Xu, H.-P. Chung, M. Parry, I. I. Kravchenko, Y.-H. Chen, A. S. Solntsev, Y. S. Kivshar, D. N. Neshev, A. A. 
Sukhorukov, Quantum metasurface for multiphoton interference and state reconstruction. Science 361, 1104-1108 (2018).

\section{Acknowledgments:}

This work was funded by the European Research Council (Grant 341054), the University of Southern Denmark (SDU2020 funding), and Villum Fonden (Grant No. 00022988). F. D. would like to thank Vladimir Zenin, Francesco Todisco, and Yun Ding for help during the optical characterization.

\section{Author contributions}

F. D. and S. B. conceived the ideas for this project. F.D. performed the numerical simulations. F. D., B. C., L. H., and X. G. designed the structure. L. H. designed the meta-hologram. B. C. and X. G. fabricated the sample. F. D set up the optical system, carried out the optical measurements and analyzed the data. F. D. and S. B. wrote the manuscript with revisions by all authors.

\section{Supplementary Materials:}

Materials and Methods

Supplementary Text

Figs. S1-S12 